\title{
Index des auteurs Author Index
}

Les chiffres se referent aux numéros des resumes. The figures refer to the abstract numbers. Adachi, M. 14, 43 Advenier, C. 155, 167 Aita, S. 43 Altieri, A. 13 Altounnyan, R.E.C. 119 Amaral-Marquez, R. 167 Aoki, H. 9, 31 Armacki, Z. 60 Arnoux, B. 3 Aubas, P. 5 Aurich, R. 180 Ayuso, R. 128

Bakran, I.jr. 142 Balog, V. 78 Balzano, G. 183 Bar-Yishay, E. 97 Basacopol, A. 132, 182 Basomba, A. 110, 128 Bavoso, P. 143 Benoist, M.R. 62, 63 Benveniste, J. 3 Bergmann, I. 113 Begogne-Bérézin, E. 176 Bersay, Cl. 39 Bierman, C.W. 175 Biot, N. 15 Blaive, B. 162 Bonini, S. 102 Bory, J. 39 Botey, J. 67 Boucaud, M. de 90 Bousquet, J. 111 Bracamonte, F. 92 Branski, H. 70 Brkic, M. 60 Bruno, G. 102 Buckley, R.H. 41 Bugnas, B. 162

Bundgaard, A. 136 Burneckis, E. 22 Bustos, G.J. 92

Caeiro, M.H. 3 Calvayrac, P. 5, 150 Canny, M. 99 Capron, A. 24 Capron, M. 24 Carantino, A. 132 Camimeo, N. 143 Carreira, J. 128 Casamitjana, M.P. 82 Cerrina, J. 3 Cesar-Ramos, J.M. 130 Charpin, J. 1 Chastang, J.F. 21 Chastagnol, D. 178 Chaussain, M. 72 Chazan, R. 184 Cheminat, J.C1. 109 Chretien, J. 173 Chu, CM. 20 Chu, J. 48

Chyrek-Borowska, S. 144 Cibas, P. 10 Clarke, C.W. 172 Clauzel, A.M. 150 Cloup, M. 56 Cocco, G. 33, 66, 121,

183 Comes, I. 32 Cooreman, J. 85 Cordier, R.J. 152 Cortes, J.L. 115 Costescu, M. 32 Craps, L. 139 Croce, J. 138 Cserháti, E. 57, 93, 94

Cvoriscec, B. 142 Czarnetzki, B.M. 35, 36,

71

D'Amato, G. 33, 66, 121,

183 Debelic, M. 76 DeBlic, J. 165 Decourt, S. 160 Delvigne, A. 45 Dervaderics, M. 104

Dessaint, J.P. 24 DeThore, J. 29 Dieguez, I. 135 DiNardo, F. 13 Di Rienzo, V. 12 Djordjevic, M. 96 Donner, C.F. 80 Dorow, P. 149 Dournovo, P. 176 Droszcz, W. 184, 185 Drouet, M. 95, 148 Dry, J. 99, 160, 174 Dujol, P. 5 Durakovic, Z. 142 Durieux, P. 178 Dziedziczko, A. 37 Elbaze, P. 39 Elliott, E.V. 25 El-Mehairy, M.M. 171 Erdos, Z. 935/894 Eseverri, J.L. 67 Esnous, D. 21

Fais, G. 12 Feldman, M. 18 Felkel, H. 134 Fernandez, M. 103

Ferreira, N.G. 130 Flouvat, B. 160 Fodor, F. 160 Fourtillan, J.B. 168 Foschino, M.P. 143

Fracchia, C. 80 Freour, P. 90 Fuchs, E. 123 Fueki, R. 9, 31 Furuya, A. 14

Gamkrelidze, A.G. 153 Garde, J. 67 Gegesi Kiss, A. 57 Georges, P. 45 Gesenhues, C. 35, 71 Geubelle, F. 61 Ghaem, A. 64 Ghory, J.E. 75 Gillissen, G. 156 Girard, J.P. 118 Godard, Ph. 5, 159 Godfrey, S. 97 Górski, P. 11 Grassi, C. 105 Greenwood, H. 161 Grilliat, J.P. 106

Grosclaude, M. 15, 166, 169 Grzelewska-Rzymowska, I.

34 Gsirishvili, M.A. 153 Guerret, M. 83 Gupta, S. 19 Gurgenidze, G.N. 153 Gyurkovits, K. 74 Hallack, G.N. 88 Haluszka, J. 70

Index des auteurs 143 
Hefny, A.M. 129 Heimlich, E.M. 118 Herman, D. 64 Hermes, H. 27 Herzog, H. 116 Higuchi, K. 100 Hofman, J. 144 Hosokawa, H. 181 Hubert, Ph. 56 Huchon, G. 178 Humbert, H. 83 Hummel, St. $16,65,101$ Hyte, M.K. 88

Ide, H. 14, 43 Ignatowicz, G. 144 Iijima, M. 14 Iikura, Y. 69 İkeda, T. 20 Ioli, F. 80 İshii, M. 68 Ishikawa, H. 133 Iwakura, M. 100

Jackson, F.A. 112 Jäger, L. 107 Jean, R. 62, 63 Johnstone, D.E. 50 Joseph, M. 24 Jovanovic, S. 96 Jung, D. 49

Kakol, J. 30 Kaveladze, A.O. 153 Kawakami, K. 69 Kawasaki, M. 44 Kelemen, J. 57, 93, 94

Kiechel, J.R. 83 Kirchhof, E. 49 Kishimoto, T. 42 Klein, G. 147 Kleinhamplova, J. 38

Kobayashi, S. 9, 31 Koda, A. 46 Kofman, J. 166, 169 Koh, Y. 14 Koizumi, S. 58 Kokubu, F. 14

Kolbas, V. 78 Kondou, T. 9

Kowalski, M.L. 34 Krasko, P. 151 Kreisler, L. 91 Kumada, S. 140 Kunkel, G. 49 Kurek, M. 30, 37, 145 Kuwabara, H. 9, 31

Labarre, Me. 39 Lallemand, A. 83 Lapalus, Ph. 162 Lavaud, J. 56 Lavène, D. 83 Lefebvre, M.A. 168 Lemoigne, F. 162 Levy, M. 97 Liccardi, G. 33 Lionnet, M.-Y. 99 Lobera, T. 135 Lokar, R. 78 Lombardo, G.G. 84 Lopes, L.S. 73

Macha, H.N. 180 Magdeleine, J. 29 Makesova, D. 26 Malunowicz, E. 185 Mancini, G. 102 Maria, Y. 5 Marin, A. 67 Marks, M.B. 81 Marland, P. 39 Marsh, D.G. 51 Martin-Mateos, Ma.A. 86 Masaki, T. 69 Mathov, E. 122 Matsui, M. 181 Matthys, H. 147 Medda, A. 13 Medici, T.C. 141 Meier-Sydow, J. 158 Meister, R. 35, 71 Melillo, G. 33, 66, 121, 183 Meriggi, A. 80 Mezei, Gy. 57, 93, 94 Michel, F.B. 5, 111, 150,159, 164 Michel, P. 21 Miguere, J. 4Mikawa, H. 87 Miskovits, G. 179 Molina, Cl. 109 Molina, M. de 82 Molkhou, P. 72 Moneo, I. 128 Montis, G. de 21, 45,

72 Mornex, J.F. 29 Morrison Smith, J. 54 Morrow Brown, H. 112 Mrzena, B. 77, 89 Mselati, J.C. 56 Mue, S. 68 Munos-Lopez, F. 86,

125 Murariu, D. 32

Nagai, H. 46 Nagakura, T. 69 Nagy, L. 154 Nakajima, H. 43 Nakajima, S. 44, 186 Nakamura, K. 46 Nakatomi, I. 46 Narimatsu, N. 43 Neffen, H. 103 Nemoto, T. 9, 31 Neukirch, F. 85 Nevot, S. 82 Nishijima, Y. 181 Nishikawa, K. 69 Nishima, S. 58 Noda, M. 43 Noguchi, H. 43 Norman, P.S. 124

Ochiai, A. 133 Oehling, A. 53, 103 Ohishi, M. 44, 186 Ohmi, T. 68 Ojkic, B. 60 Okada, T. 14

Orehek, J. 1 Orr, T.S.C. 7 Ouechni, M.M. 168 Oury, M. 163

Pacheco, Y. 15 Padegimas, B. 10

Palma-Carlos, A.G. 73,

126, 130, 146 Palma-Carlos, G. 73 Patriarca, G. 12 Paupe, J. 55, 83, 98, 131,

165 Pepys, J. 108 Perdrizet, S. 85 Perrin-Fayolle, M. 15,

166, 169 Pësic, V. 60, 96 Petrû, V. 77 Pierre, J. 176 Piotrowska, B. 185 Pitzalis, F. 13 Plaza, A.M. 82 Póder, Gy. 57, 93, 94 Pompidou, A. 21 Popescu, C. 132, 182 Popescu, I. Gr. 32 Porru, F. 13 Pradalier, A. 99, 160 Prazakova, J. 134 Prenner, B.M. 88

Racineux, J.L. 163 Radielovic, P. 141 Rebohle, E. 27 Resta, O. 143 Revenda, M. 77 Ribeiro, L.N. 130 Ricciotti, F. 13 Romano, A. 12 Romanski, B. 30, 37, 145 Rotigliano, G. 102 Rozniecki, J. 11, 34 Rudolph, R. 49 Ruff, F. 117, 165, 167, 177, 178 Rufin, P. 62,63, 131

Sabbah, A. 95, 148 Sakakibara, H. 133 Santais, M.C. 167, 178 Santana, P.R.F. 146 Sanz, M.L. 103 Sasaki, S. 100 Sasaki, Y. 43 Savic, Lj. 60 
144

Resumes du Xe Congrès d'Interasma, Paris 1981

Schiano, M. 33,66, 183 Schiavino, D. 12 Scheinmann, P. 55, 83,

165 Schino, P. 143 Schmidt, A. 136 Schroder, H. 40 Scislicki, A. 70 Seek, J. 170 Sehon, A.H. 127 Seropian, E. 6 Seyrig, C. 161 Shiho, O. 42 Shimada, T. 69 Sierra, J.I. 82 Sladek, M. 49

Slapke, J. 16, 65, 101 Soyinka, F. 114 Spada, E. 80 Spicák, V. 77, 89 Stam, J. 137 Stefanovic, M. 96 Sudo, M. 181

Suemura, M. 42 Suetsugu, S. 133 Sugisaki, T. 14, 43 Sunaga, Y. 9,31 Suzuki, I. 79 Swiatkowski, M. 30, 37,

145 Szekeres, I. 74 Szentivanyi, A. 2 Szentivanyi, J. 2 Szmidt, M. 11, 34 Szüle, P. 104

Tabart, J. 39 Taïb, J 5

Takahashi, T. 14, 43 Takagi, O. 44, 186 Takise, A. 9 Takishima, T. 68 Tamura, G. 68 Tanaka, T.

9 Tateno, K. 79 Tesic, R. 96

Toccaceli, F. 102 Tomioka, S. 31 Tomita, K. 20 Tonnel, A.B. 24 Torre Morin, F. de la 59 Tóth,

A. 104, 154, 179 Touraine, J.-L. 17 Trindade, J.C. 130 Troussier, J. 163 Tsubomizu, T. 14, 43

Tsuya, Y. 44, 186 Tuchais, E. 163

Ueno, H. 186 Ulmeanu, V. 32 Umeda, H. 133 Uryniak, T.J. 88 Uvnäs, B. 8 Uzzan, B. 173

Vacca, F. 102

Vargas Cornelio, S. 28, 47

Vecchio, C. 80 Venuti, A. 12 Vergnon, J.M. 15 Vervloet, D. 120 Vialatte, J. 52,98, 131 Volozni, D. 97 Vondra, V. 134 Vonlanthen, M.C. 62, 63, 165 Vyhnálek, M. 77

Wallenstein, G. 27 Wandell, M. 161, 170 Wasserman, S.I. 23 Werninghaus 157 Willim, G 70

Yabuuchi, H. 20 Yamamura, Y. 42 Yokokawa, T. 43

Zhu, W.R. 48 